\title{
APPLICATION OF OPTICAL FLOW FOR RIVER VELOCIMETRY
}

\author{
Musaab Khalid $^{l} \quad$ Lionel Pénard ${ }^{l} \quad$ Etienne Mémin ${ }^{2}$ \\ ${ }^{1}$ Irstea, UR HHLY, centre de Lyon-Villeurbanne, F-69616 Villeurbanne, France \\ ${ }^{2}$ Inria, Campus universitaire de Beaulieu, F-35042 Rennes Cedex, France
}

\begin{abstract}
We present a new method for the application of imagebased river velocimetry. Our method is dedicated to high velocity river flows and it is based on an optical flow scheme. Unlike traditional Particle Image Velocimetry (PIV) methods, optical flow provides dense estimations and it is highly adaptable to different applications. We therefore propose a new physics-based optical flow derived from scalar transport equation and augmented with a weighted diffusion term. The results are evaluated on a Lagrangian basis by means of trajectories reconstruction. The trajectories of few passive particles of interest are reconstructed, based on the successive estimated vector fields. Our method outperformed traditional PIV-based and original optical flow methods.
\end{abstract}

Index Terms- Optical flow, river velocimetry, $\mathrm{PIV}$, image sequence processing

\section{INTRODUCTION}

Estimating velocities of moving objects using image sequences is an old (but still ongoing) area of research and it serves many applications. In river velocimetry, one technique stands out due to the seminal work of Fujita et al [1], short-named LSPIV (Large-Scale Particle Image Velocimetry). Based on PIV [2], this technique establishes correspondences between windows in successive ortho-rectified images, enabling the recovery of real world velocity vector fields. PIV despite its popularity in fluids research is limited in many aspects. Firstly, it favors well-seeded flows. Otherwise, it is difficult to compute unique correlation peaks between tracked image windows. In addition, choosing a suitable window size is important but also a tricky task. One needs a bigger window to compute better correlation score but then a bigger window might contain multiple motions. Furthermore, PIV is not based on any physical principle that is related to fluids nature. Optical flow on the other hand showed great potential to replace PIV. Introduced first in computer vision community [3], optical flow is a well-developed image- based velocity estimation technique. It minimizes an energy function that consists of data and regularization terms. The data term is based on the brightness constancy assumption, i.e. it is supposed that points keep their color intensity value while moving in a small time interval. The regularization term serves to impose spatial coherency constraints on the recovered vector field. A strictly positive weighting parameter is introduced in-between to determine the importance of one term against the other. Unless a very smoothed vector field is needed, where in this case a large value for this parameter is used, it is not in general an issue to find a suitable value. The regularization term permits adding a priori information about the expected vector field. This turns out to be of great importance in poorly seeded areas. It helps fill-in where no motion could be observed. Contrary to PIV, optical flow produces dense velocity vector fields. This is generally a desired feature for vector field analysis when one aims for instance at extracting vorticity fields or mixing criterion. The variational formulation permits high adaptability to different situations. Image restoration for instance has a similar structure to optical flow but it is a totally different application.

\section{OPTICAL FLOW}

The brightness constancy assumption leads to the wellknown optical flow constraint equation OFCE:

$$
\frac{\partial I}{\partial t}+\nabla I . w=0
$$

where $\nabla I=\left[I_{x}, I_{y}\right]^{T}$ is the spatial gradient of the image intensity function $I$, the subscripts denote partial derivatives and $w=[u, v]^{T}$ represents the velocity components. It is an ill-posed problem since we have only one equation in two unknowns. One could also see that in the case of zero gradient (uniform image intensity values), an infinite set of $w$ would satisfy OFCE. This underdetermined system is solved by adding another term in form of regularization to make the problem well-posed. The regularization term also helps avoiding spurious solutions when the gradient vanishes. Horn and Schunck (HS) [3] proposed to minimize the norm of velocity gradient $\nabla w$ as 
regularization. This imposes that the velocity vector field should vary smoothly on the entire image. In other words, this states that neighboring points tend to move together. The final energy function reads:

$$
\int_{\Omega}\left(\frac{\partial I}{\partial t}+\nabla I . w\right)^{2}+\alpha\|\nabla w\|^{2} d x
$$

where $\alpha$ is a strictly positive weighting factor. Many improvements to the original model have been proposed over the years to deal with different possible violations. Quadratic functions for example, known for their sensitivity to outliers, were replaced by robust functions for more robustness [4]. Furthermore, the above linear model assumes small displacements. A coarse-to-fine strategy is utilized to deal with large displacements. Simply put, at the coarsest level, only a small displacement is sought and the vector field is then propagated to the finer level where only small increment field is estimated. This process is repeated until the finest level (original image). However optical flow was mostly developed for image sequences involving rigid motions and not necessarily directly applicable to fluids. In recent years it has been also adopted in experimental fluid mechanics. New physicsbased data terms and/or regularizers have been thus derived for the special case of fluids motions (See [5] for a review).

\section{SGSD MODEL}

Liu et al [6] formally established the relationship between optical flow and fluid flow. Based on the perspective projection of the transport equation on the 2D plane, optical flow is proportional to the pathaveraged velocity field weighted with a relevant field quantity. This gave the physical foundation needed since the brightness constancy assumption is not based on any physical principle.

Following Cassisa et al [7] and Chen et al [8], we propose a data term based on Large Eddy Simulation (LES) decomposition [9] of the transport equation. We assume that the image intensity function $I$ is related to some passive scalar field concentration $C$. The idea is to model small scales contributions that are normally filtered out during image acquisition process which only captures contributions larger than image pixel size. The scalar transport equation reads:

$$
\frac{\partial \bar{C}}{\partial t}+\nabla \cdot(\overline{C w})-\frac{1}{R e S c} \Delta \bar{C}=0
$$

where $\nabla$. represents the divergence, $\Delta$ is the Laplacian while the upper bar designates a filtered term. $R e$ and $S c$ are Reynolds number and Schmidt number, respectively. The filtered non-linear term $\overline{C w}$ could be rewritten as resolved and unresolved parts: $\overline{C w}=\bar{C} \bar{w}+$ $\tau$ which leads to:

$$
\frac{\partial \bar{C}}{\partial t}+\nabla \cdot(\bar{C} \bar{w})+\nabla \cdot \tau-\frac{1}{\operatorname{ReSc}} \Delta \bar{C}=0
$$

Authors in [7] considered $\tau$ to be related to turbulent viscosity $\tau=-D_{t} \nabla \bar{C}$ where $D_{t}$ is a turbulent diffusion coefficient and suggested a model to estimate it, while in [8] authors took a different route and proposed to model $\tau$ directly. Both models considerably improved the results. For high velocity river sequences, we opted for a simpler derivation to estimate $D_{t}$ based on Prandtl mixing-length model. This model uses the stream-wise velocity $u$ and the mixing length $l$ to estimate the turbulent viscosity $v_{t}=l^{2}$. $\left|\frac{d u}{d y}\right|$. Turbulent viscosity is relevant to the computation of $D_{t}$ as they are related by $D_{t}=\frac{v_{t}}{s c_{t}} \quad$ where $s c_{t}$ is the turbulent Schmidt number (different from above mentioned Schmidt number $S c$ ) that has an empirical value normally determined experimentally. It has been reported that $s c_{t}$ has widespread values between 0.2 and 3 [10] while others suggested 0.85 to be optimal for water flows. If we take it to be 1.0 then $D_{t}$ is equal to $v_{t}$. The mixing length $l$ is also considered unity because optical flow modeling is differential in nature and assumes small motions that may not be more than one pixel (coarse-to-fine, graduated non-convexity and warping) [11][12]. To estimate $D_{t}$, we take advantage of the pyramidal coarseto-fine strategy to get initial estimates for the velocity. Such scheme introduces an estimation procedure organized along a multiresolution pyramid of image data. At the coarser level a first estimate can be easily provided. The molecular diffusion term $\frac{1}{\operatorname{Re} S c} \Delta \bar{C}$ in equations (3) and (4) is generally neglected. Another assumption is made that the incompressibility condition for water still holds on the 2D plane, no motion thus on the third direction is considered. Applying the incompressibility condition and substituting $\tau$, equation (4) becomes:

$$
\frac{\partial \bar{C}}{\partial t}+\nabla \bar{C} \cdot \bar{w}-D_{t}(\Delta \bar{C})=0
$$

One can observe that the final equation (5) is equivalent to the traditional brightness constancy assumption (1) plus a new term $D_{t}(\Delta \bar{C})$, which shows that the traditional optical flow data term already considers the incompressibility condition and that it is physically consistent with the observed fluid. The new term is nothing but a weighted diffusion term. In practice we use inhomogeneous diffusion coefficients by considering the mixed length model also for $v$.

For the regularization part, Corpetti et al [13] showed that the traditional regularization term may not be suitable for fluids in general as it tends to equally penalize divergence and vorticity. However in the case of no or negligible vorticity and incompressible fluids like in rivers, such regularization seems plausible. Firstly, penalizing the divergence is desirable to enforce the incompressibility. Secondly, penalizing the vorticity is also beneficial, considering that these image 


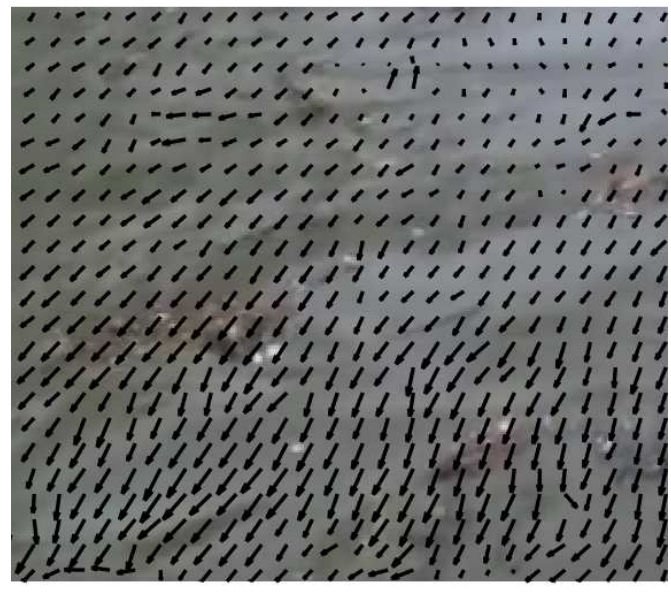

(a) "Gaves de Pau" sequence, SGSD velocity field.

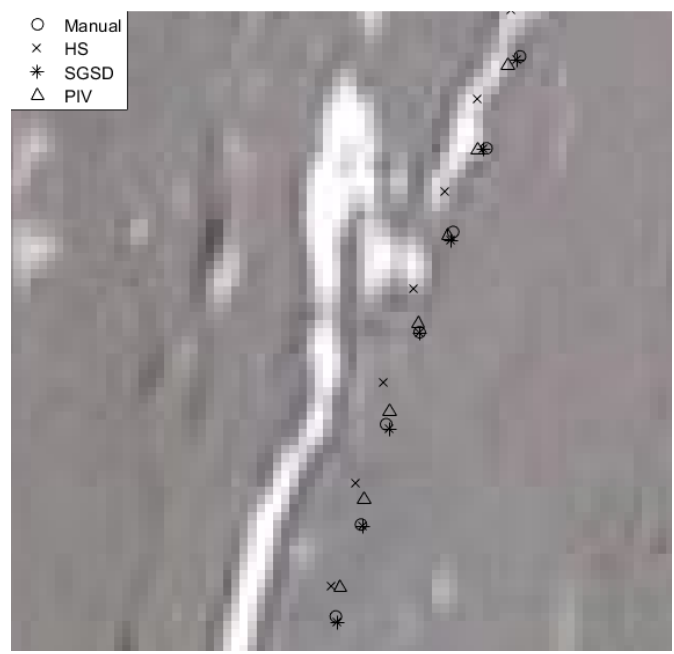

(c) "Arc" sequence, middle of the trajectories

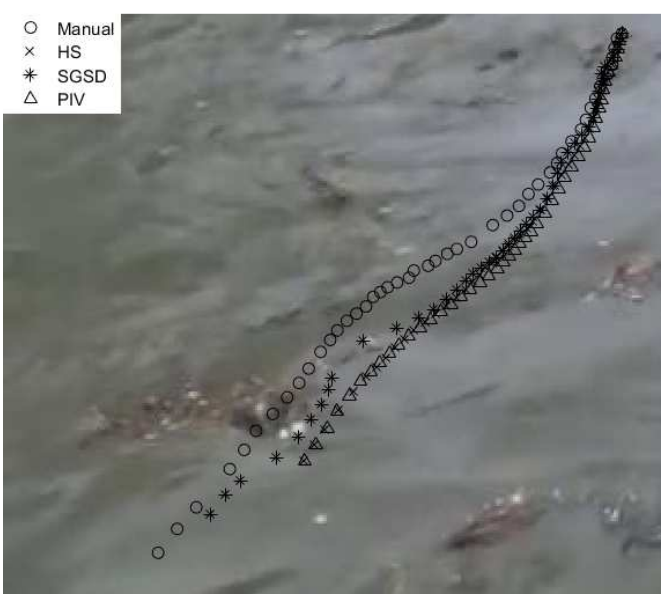

(b) “Gaves de Pau” sequence.

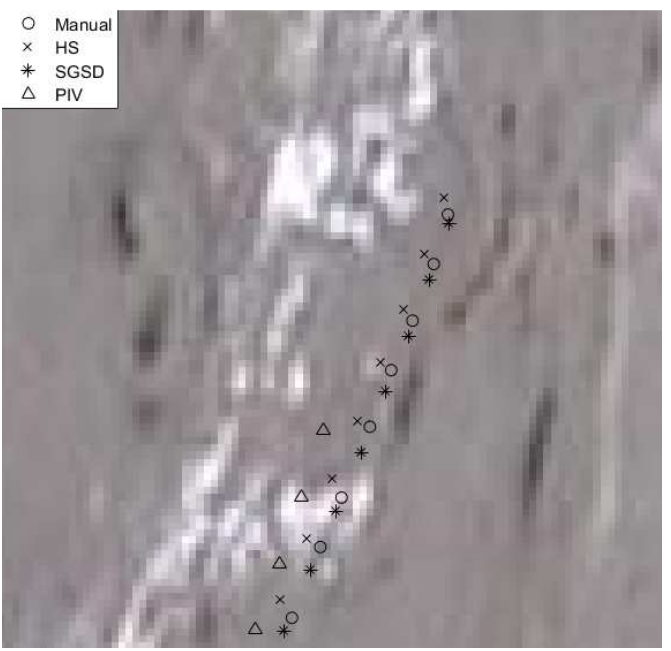

(d) "Arc" sequence, end of the trajectories

Figure 1: Trajectories reconstructed using different methods and superposed on the first image of each sequence. (b). velocity plot of one SGSD estimation superposed on the first image of "Gaves de Pau" sequence.

sequences exhibit more translational patterns than rotational ones. These translational patterns do not promote chaotic motions (provided that no out-of-plane components are observed); the notion of pixels moving together, imposed by this regularization is still valid. In high velocity river sequences, it is unlikely to obtain a uniform intensity zone locally while having very different velocity vectors, more likely a mixing phenomenon will occur which will cause image intensity perturbations. In this latter case it is the data term that is dominant and guides the optimization. The same logic could be applied backwards, having uniform and smooth intensity is likely due to similar velocity vectors. It is in uniform intensity zones where regularization importance prevails due to the vanishing image gradient. Hence, this regularization is very convenient in this case.

In many cases the brightness constancy assumption is violated due to light changes or occlusions to cite a few. This is even worse in such image sequences because sources/sinks in the flow may cause parts of the flow to appear/disappear on the imaged surface at consecutive time steps. The data term hence must be relaxed to accept outliers by using robust penalization. However, we use quadratic penalization for the regularization term because it promotes smooth vector fields. Even if most of these sequences are turbulent in a sense, they do not normally exhibit sudden change of velocity directions or magnitude. Using robust penalization for the 
regularization may introduce non-desired fictional discontinuities. The final optical flow model reads:

$$
\int_{\Omega} \psi\left(\frac{\partial I}{\partial t}+\nabla I \cdot w-D_{t}(\Delta I)\right)+\alpha\|\nabla w\|^{2} d x
$$

where $\psi$ is a robust function such that $\psi(x)=$ $\sqrt{x^{2}+\epsilon^{2}}$ and $\epsilon$ is a small fixed positive constant that assures the function differentiability, typically set to 0.001. We refer to our method as SubGrid Scale Diffusion (SGSD).

\section{RESULTS AND DISCUSSION}

In comparison to rigid motion optical flow, it is difficult to evaluate velocity estimations applied to image sequences of natural phenomena directly in image space. To generate a ground truth for such cases, the physical phenomenon should be simulated while taking into account all the factors and physical processes that contribute to its occurrence. We propose to evaluate the estimated vector fields on a Langragian basis by the way of trajectories reconstruction. Using Runge-Kutta $4^{\text {th }}$ order integration method, trajectories of few passive particles of interest are reconstructed based on their successive estimated velocity. These are then compared to their manually-reconstructed counterparts. We show the trajectories of different methods alongside the manual trajectory to visually evaluate the results. Our model clearly improved the results over traditional optical flow and PIV-based method that produces dense estimations). The "Gaves de Pau" sequence in Figure 1 (b) is very turbulent and contains 3D motions where the water surface goes down and up throughout the sequence. In addition, it contains many zones with uniform intensity. The result on this sequence highlights the merits of the additional diffusion term. Due to significant velocity gradients, the contribution of the diffusion term is increased. Consequently, SGSD was able to recover better velocity magnitude and direction. The "Arc" sequence in Figure 1(c), (d) is less noisy than previous sequence. We track a particle in a well-seeded area and we see that SGSD is always superior in (c) which shows the middle of the trajectory and in (d) which shows the end of the trajectory.

\section{CONCLUSION}

Optical flow proved to be very attractive for imagebased velocity estimation in hydraulics and experimental fluid mechanics research. We proposed a new optical flow model based on subgrid modeling that adds a weighted diffusion term. The model improved the results and outperformed traditional PIV and original optical flow methods (HS). Due to its flexibility to accept new physics-based models, it could be applied to a wide variety of applications.

\section{REFERENCES}

[1] I. Fujita, M. Muste, and A. Kruger, "Large-Scale Particle Image Velocimetry for Flow Analysis in Hydraulic Engineering Applications," Journal of Hydraulic Research, vol. 36, no. 3, pp. 397-414, 1998.

[2] R. J. Adrian, "Particle-Imaging Techniques For Experimental Fluid-Mechanics," Annual Review of Fluid Mechanics, vol. 23, no. 1, pp. 261-304, Jan. 1991.

[3] B. K. P. Horn and B. G. Schunck, "Determining optical flow," Artificial Intelligence, vol. 17, no. 1-3, pp. 185203, Aug. 1981.

[4] M. J. Black and P. Anandan, "The Robust Estimation of Multiple Motions: Parametric and Piecewise-Smooth Flow Fields," Computer Vision and Image Understanding, vol. 63, no. 1, pp. 75-104, Jan. 1996.

[5] D. Heitz, E. Mémin, and C. Schnörr, "Variational fluid flow measurements from image sequences: synopsis and perspectives," Experiments in Fluids, vol. 48, no. 3, pp. 369-393, Mar. 2010.

[6] T. Liu and L. Shen, "Fluid Flow And Optical Flow," Journal of Fluid Mechanics, vol. 614, pp. 253-291, Nov. 2008.

[7] C. Cassisa, S. Simoens, V. Prinet, and L. Shao, "Subgrid scale formulation of optical flow for the study of turbulent flow," Experiments in Fluids, vol. 51, no. 6, pp. 1739-1754, Dec. 2011.

[8] X. Chen, P. Zillé, L. Shao, and T. Corpetti, "Optical flow for incompressible turbulence motion estimation," Experiments in Fluids, vol. 56, no. 1, Jan. 2015.

[9] C. L. Ribault, S. Sarkar, and S. A. Stanley, "Large Eddy Simulation of Evolution of a Passive Scalar in Plane Jet," American Institute of Aeronautics and Astronautics Journal, vol. 39, no. 8, pp. 1509-1516, Aug. 2001.

[10] Y. Tominaga and T. Stathopoulos, "Turbulent Schmidt numbers for CFD analysis with various types of flowfield," Atmospheric Environment, vol. 41, no. 37, pp. 8091-8099, Dec. 2007.

[11] D. Sun, S. Roth, and M. J. Black, "A Quantitative Analysis of Current Practices in Optical Flow Estimation and the Principles Behind Them," International Journal of Computer Vision, Jan. 2014.

[12] T. Brox, A. Bruhn, N. Papenberg, and J. Weickert, "High Accuracy Optical Flow Estimation Based on a Theory for Warping," in Computer Vision - ECCV 2004, 2004, vol. 3024, pp. 25-36.

[13] T. Corpetti, D. Heitz, G. Arroyo, E. Mémin, and A. Santa-Cruz, "Fluid experimental flow estimation based on an optical-flow scheme," Experiments in Fluids, vol. 40, no. 1, pp. 80-97, Oct. 2005. 